\title{
Cold scissors ploughing technique in hysteroscopic adhesiolysis: a comparative study
}

\author{
Xingping Zhao, Aiqian Zhang, Bingsi Gao, Arvind Burjoo, Huan Huang, Dabao Xu \\ Department of Gynecology, Third Xiangya Hospital of Central South University, Changsha 410013, China \\ Contributions: (I) Conception and design: D Xu; (II) Administrative support: None; (III) Provision of study materials or patients: D Xu; (VI) \\ Collection and assembly of data: X Zhao; (V) Data analysis and interpretation: X Zhao, A Zhang, B Gao; (VI) Manuscript writing: All authors; (VII) \\ Final approval of manuscript: All authors. \\ Correspondence to: Dabao Xu, MD. Department of Obstetrics and Gynecology, Third Xiangya Hospital of Central South University, 138 Tongzipo \\ Rd., Changsha 410013, China. Email: dabaoxu@yahoo.com.
}

\begin{abstract}
Background: Intrauterine adhesions (IUAs) can be dissected using hysteroscopic scissors (cold scissors) or other methods, but there is no consensus on which hysteroscopic method is preferable. There is also no consensus on the method of how to deal with the scar tissue on the surface of the intrauterine cavity.

Methods: From January 2016 to October 2017, 179 patients who had HA met the enrollment criteria (see the text below), and their data were analyzed retrospectively. In addition, all patients were divided into three groups according to the surgical techniques used. The groups were the ploughing group (PG) (using cold scissors to dissect the adhesion and cut the scar tissue using a ploughing technique) (n=81), the traditional group (TG) (using cold scissors to dissect the adhesion, but not deal with the scar tissue) (n=42), and the electrosurgical group (EG) (using a resectoscope to dissect the adhesion with an energy L-hook electrode, and not deal with the scar tissue) ( $\mathrm{n}=56)$. Safety (surgical complications), feasibility (surgical technique replacement rate), and postoperative efficacy (reduction of AFS score, pregnancy, and live birth rate), were each evaluated between groups.
\end{abstract}

Results: No statistically significant differences between the groups were observed in basic preoperative information $(\mathrm{P}>0.05)$, while there were significant differences between PG and TG, as well as PG and EG in postoperative AFS scores (PG vs. TG: $\mathrm{P}=0.007$; $\mathrm{PG}$ vs. $\mathrm{EG}: \mathrm{P}<0.001)$ and pregnancy outcome $(\mathrm{PG}$ vs. TG: $\mathrm{P}=0.039$; $\mathrm{PG}$ vs. $\mathrm{EG}$ : $\mathrm{P}<0.001)$. No patients had surgical complications such as uterine perforations, moderate or severe fluid overload, heavy uterine bleeding, nor any surgical technique replacements (for example, transfer to use a resectoscope).

Conclusions: Cold scissors ploughing technique in HA is effective, feasible, and safe, and thus worthy of further study.

Keywords: Intrauterine adhesion (IUA); hysteroscopic adhesiolysis; scar tissue; cold scissors; technique

Submitted Aug 08, 2019. Accepted for publication Oct 21, 2019.

doi: $10.21037 /$ atm.2019.11.136

View this article at: http://dx.doi.org/10.21037/atm.2019.11.136

\section{Introduction}

Intrauterine adhesion (IUA), also called Asherman syndrome, was first described and reported by Joseph Asherman in 1948 (1). Adhesions are believed to occur when contiguous traumatized uterine walls heal by abnormal fibrous secondary to trauma to a gravid, or non-gravid uterine cavity. Postpartum curettage and abortion were considered the major cause of IUA. Other causes include genital tuberculosis, pelvic irradiation, and uterine surgery (including hysteroscopic surgery) (2). It was inevitable that direct contact of these abraded areas where denudation or perforation of the myometrium would result in their coalescence by scarring (3). The longer the course of the 


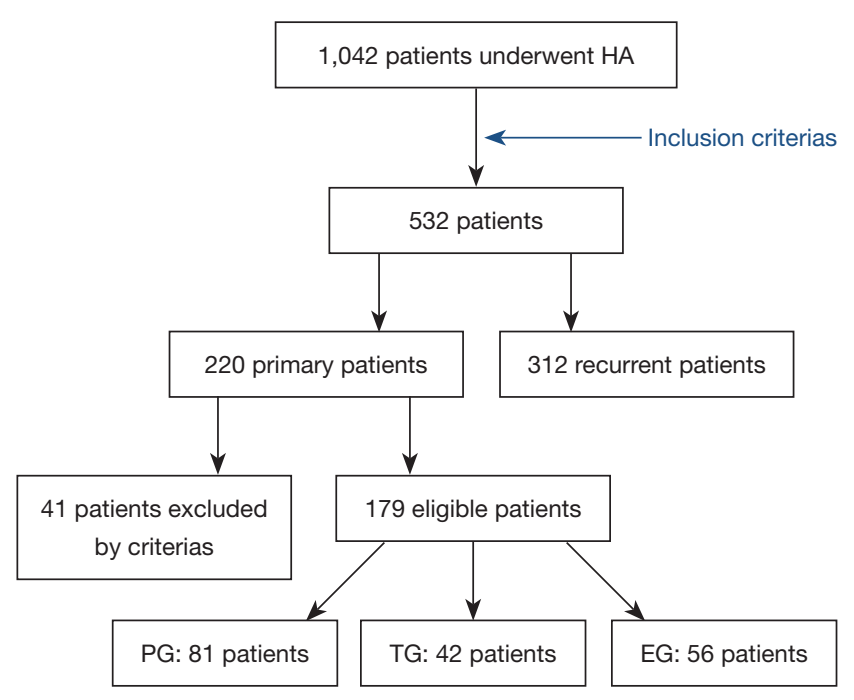

Figure 1 Flowchart of the study population. PG, ploughing group; TG, traditional group; EG, electrosurgical group.

disease, the denser the adhesion (4). IUA typically presents with amenorrhea, hypomenorrhea, infertility, and repeated abortions $(5,6)$. Diagnosis and treatment outcomes have been improved dramatically, as hysteroscopic techniques have developed (7).

IUAs can be dissected using hysteroscopic scissors (cold scissors) or other methods, such as the L-hook electrode and loop electrode. HA has been the gold treatment for IUA (8). Most of the currently published papers describe HA using electrical instruments, which may cause thermal injury to the endometrium $(9,10)$. Previously, we reported using the HA ploughing technique with cold scissors for the first time in the literature in 2015 (Zhang et al.) (11). However, there is no consensus on which hysteroscopic method is preferable: energy-based using electrodes or mechanically based using cold scissors. The scar tissue on the surface of the intrauterine cavity is a critical issue as it might prevent the blood supply going to the surface of the cavity for the endometrium and cause the uterine cavity to shrink as well. There is also no consensus on the method of how to deal with the scar tissue on the surface of the intrauterine cavity, and it is unclear whether it is better to cut the scar into several parts using a ploughing technique, resect, and remove the scar tissue, or just leave the scar tissue alone. Until now, no large cases studies have stated the efficacy, feasibility, and safety of HA using the cold scissors ploughing technique, nor have there been any comparative studies between HA techniques with or without managing the scar tissue on the surface of the uterine cavity. This study aimed to assess the efficacy, feasibility, and safety of HA using cold scissors technique.

\section{Methods}

\section{Patients}

A total of 532 patients had undergone HA met the inclusion criteria at the Third Xiangya Hospital of Central South University between July 2015 and July 2017. Written informed consent was signed voluntarily by patients, and the study was approved by the ethics committee. IUAs were scored by the same surgeon, according to the American Fertility Society (AFS) classification system (12). IUAs were scored as follows: 1-4 (mild), 5-8 (moderate), and 9-12 (severe).

The inclusion criteria included the following: (I) IUA confirmed by hysteroscopy and undergoing HA first time at our hospital; (II) desire for fertility; (III) age between 1840 years; (IV) successful follow-up to 2 years after surgery; (V) complete data and information of hospitalization, particularly clear records of surgical methods; (VI) normal endocrine function and ovulation; (VII) all hysteroscopies were done by experienced experts. Exclusion criteria included the following: (I) recurrent IUA; (II) tubercular IUA; (III) presence of other intrauterine diseases such as endometrial polyps or atypical hyperplasia; (IV) bilateral blocked tubes post-hysteroscopic surgery; (V) grossly abnormal partner semen; (VI) severe systemic disease or contraindications to estrogen or surgery; (VII) both tubal ostia were not exposed postoperatively

Finally, 179 eligible IUA patients were selected retrospectively according to the inclusion criteria and exclusion criteria in our study. Three groups were formed according to the HA operation methods: the cold scissor ploughing group (PG) ( $\mathrm{n}=81$ cases), the traditional cold scissor technique group (TG) ( $\mathrm{n}=42$ cases), and the electrosurgical group (EG) (n=56 cases) (Figure 1).

\section{Surgical procedure}

HA was performed under intravenous anesthesia. All patients fasted for 6-8 hours before surgery. Rectal misoprostol (400 mg) was administered 2 hours before surgery. A sterile saline solution was used to distend the uterus. Distension pressure was set to $110-120 \mathrm{mmHg}$ with a flow rate of $300-350 \mathrm{~mL} / \mathrm{min}$. A diagnostic hysteroscopy 

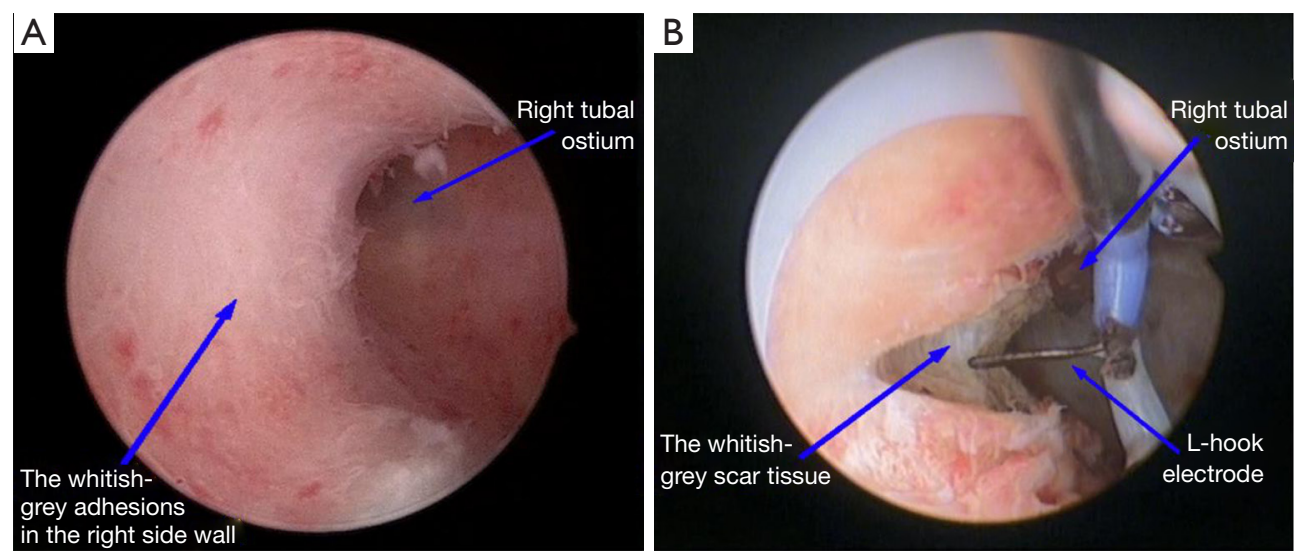

Figure 2 EG: the whitish-grey scar tissue covered the right-side wall (A). L-hook electrode was used in HA (B). EG, electrosurgical group.
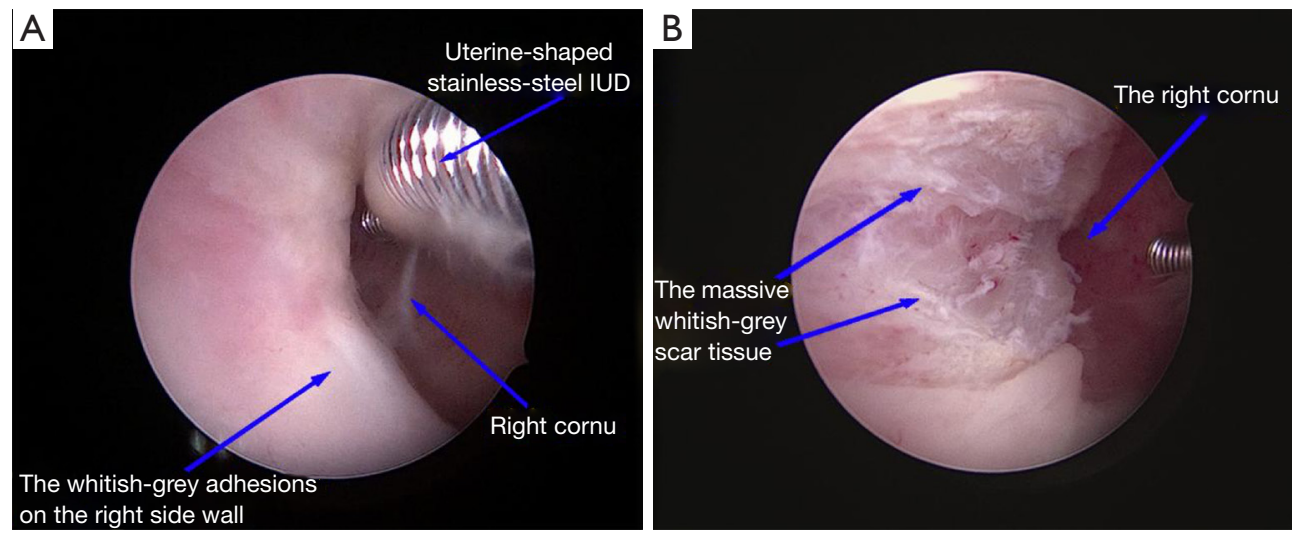

Figure 3 TG: the whitish-grey adhesions in the right-side wall in preoperative (A). Dissected the adhesion by cold scissors without dealing with the scar tissue (B). TG, traditional group.

with an out sheath of $4.5 \mathrm{~mm}$ in diameter was used to explore the uterine cavity and evaluate the AFS scores of adhesions prior to perform HA. The operation was monitored under transabdominal sonographic guidance.

(I) For EG, the cervix was gradually dilated to 9 to $9.5 \mathrm{~mm}$. An 8.5 - $\mathrm{mm}$ outer sheath bipolar resectohysteroscope with an L-hook electrode was inserted into the uterine cavity. The adhesions of the uterine side wall were separated by the L-hook electrode (with minimal power energy) from the fundus to internal cervical orifice; the adhesions of the fundus were transversely separated from the midpoint of the fundus to the left and right corners. Complete adhesiolysis was defined as recovery of the normal shape of the uterine cavity with clearly visible tubal ostium (Figure 2).
(II) For TG, a $6.5 \mathrm{~mm}$ operative hysteroscope was used to perform HA after the cervical canal was dilated to $7.5 \mathrm{~mm}$. The adhesions in the uterine cavity were separated by a 7 Fr rigid single action scissors until the normal uterine cavity was retrieved. The adhesions located in the central part of the uterine cavity were usually dissected firstly and then the lateral adhesions. Tubal ostia should be retrieved as possible firstly in patients whose ostium/ostia cannot be visualized because of the adhesions after the anatomical marker (tubal ostium/ostia) were successfully visualized, then the adhesions could be easily dissected. A 5 Fr bipolar electrode pole was used for hemostasis only in patients with substantial bleeding during adhesiolysis (Figure 3).

(III) For PG, a $6.5 \mathrm{~mm}$ operative hysteroscope was 

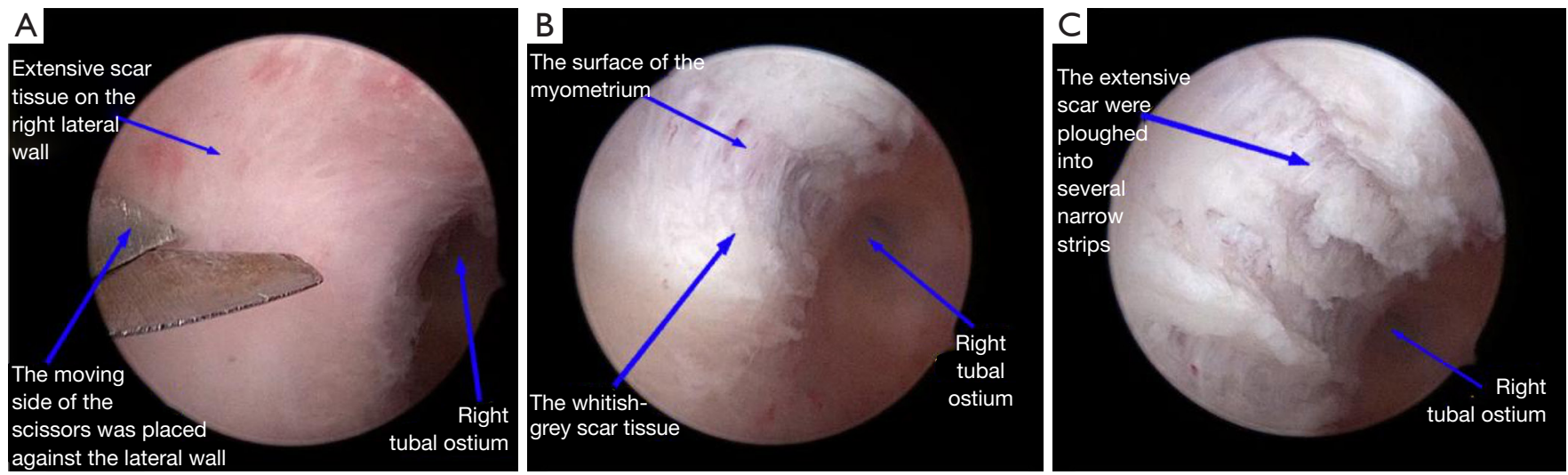

Figure 4 PG: the scissors were placed against the right lateral wall of the uterine cavity to dissect the extensive scars (A). Ploughed through the scar, cutting through to the depth of the surface of the myometrium (B). The extensive scars were ploughed into several longitudinal narrow strips (C). PG, ploughing group.

used to perform HA after the cervical canal was dilated to $7.5 \mathrm{~mm}$. The adhesions in the uterine cavity were separated by a 7 Fr rigid single action scissors until the normal uterine cavity was retrieved. Additionally, the scar tissue covering the anterior and posterior walls of the uterine cavity was ploughed longitudinally into several narrow strips (11). We ploughed through the scar, cutting through to the depth of the surface of the myometrium. Myometrium was pink in color compared to the white-colored, dense scar tissue. This ploughing technique opened the uterine cavity by loosening the walls from the contraction of the scarred tissue. Moreover, the ploughing provided a fresh, rich blood-supplied surface on which the endometrium could grow and cover after the surgery. When performing this step, more attention must be taken not to damage the underlying myometrium, which might lie under the scars (Figure 4).

After the entire uterine cavity was opened successfully with clearly visible fallopian tubal ostia. A uterine-shaped stainless-steel IUD was inserted into the uterine cavity with its position checked via hysteroscopy to ensure that the size of the IUD matched the uterine cavity size and that the IUD was correctly positioned. A doublechannel, 12-Foley catheter balloon, with the top catheter portion removed, was inserted into the uterine cavity and distended using $2.5 \mathrm{~mL}$ of sterile saline with the balloon in the center of the uterine-shaped IUD. Next, $3 \mathrm{~mL}$ hyaluronic acid gel was injected into the uterine cavity through the catheter (5). Complications and the exchange of surgical techniques would be recorded.

\section{Postoperative management and follow-up}

The Foley catheter was removed after 3 days for moderate IUAs and 7 days for severe IUAs postoperatively. All patients had an ultrasound scan to ensure the position of the IUD was normal after removing the catheter. Hormone therapy began with estradiol valerate $3 \mathrm{mg}$ Bid for 21 days or following their menstrual cycle and added progesterone $100 \mathrm{mg}$ Qn for the last 6 days of the menstrual cycle to promote endometrial growth for three cycles. For severe adhesions, the first following up hesteroscopy was performed after the first menstruation postoperative, and the second following up hesteroscopy was 3 months later. For moderate adhesions, hysteroscopy was performed after the third menstruation postoperatively. Hysteroscopic tubal catheterization and hydrotubation were used to evaluate tubal patency if no adhesion was found in following up hysteroscopy. But if adhesions had returned, an adhesiolysis procedure was repeated until further improvement is likely impossible. The IUD was removed during the final hysteroscopy. The menstrual volume, AFS scores, and size, and shape of the uterine cavity were updated once again during the hysteroscopy of the first time for moderate IUAs and the second time for severe IUAs postoperative.

Patients were suggested to resume their conception efforts after successful HA. All patients were followed-up for at least 2 years of the postoperative period for pregnancy and pregnancy outcomes. 
Table 1 General preoperative information

\begin{tabular}{|c|c|c|c|c|c|}
\hline Variable & $P G(n=81)$ & $\mathrm{TG}(\mathrm{n}=42)$ & $E G(n=56)$ & P1 (PG vs. TG) & P2 (PG vs. EG) \\
\hline AFS score (mean $\pm \mathrm{SD})$ & $8.48(1.73)$ & $8.74(1.19)$ & $8.21(1.64)$ & 0.392 & 0.366 \\
\hline Gravidity, n (\%) & & & & 0.458 & 0.930 \\
\hline$\leq 2$ & $38(46.9)$ & $16(38.1)$ & $25(44.6)$ & & \\
\hline Abortion, n (\%) & & & & 0.654 & 0.911 \\
\hline$\leq 2$ & $52(65.4)$ & $25(59.5)$ & $38(679)$ & & \\
\hline$>2$ & $28(34.6)$ & $17(40.5)$ & $18(32.1)$ & & \\
\hline \multicolumn{2}{|c|}{ History of uterine cavity operation, $\mathrm{n}(\%)$} & & & 0.466 & 0.526 \\
\hline Other hysteroscopic surgeries & $10(12.3)$ & $4(9.5)$ & $7(12.5)$ & & \\
\hline Unknown & $1(1.2)$ & $0(0.0)$ & $0(0.0)$ & & \\
\hline Degree of adhesion, n (\%) & & & & 0.824 & 0.440 \\
\hline Moderate adhesion & $47(58.0)$ & $34(61.9)$ & $37(66.1)$ & & \\
\hline Severe adhesion & $34(42.0)$ & $16(38.1)$ & 19 (33.9) & & \\
\hline
\end{tabular}

D\&C, dilation and curettage; PG, ploughing group; TG, traditional group; EG, electrosurgical group.

\section{Statistics}

R Studio 1.1.442 was used for data analysis. Numerical variable data were expressed by mean \pm standard deviation $\bar{x} \pm s)$. Variable rates and composition ratios were expressed. The $t$-test and chi-square test were adopted, and $\mathrm{P}<0.05$ was considered as statistically significant.

\section{Results}

\section{General preoperative information}

The mean $( \pm \mathrm{SD})$ ages of women at the time of initial adhesiolysis were $30.84 \pm 4.19,30.33 \pm 4.37$, and $31.77 \pm$ 5.18 years for $\mathrm{PG}, \mathrm{TG}$, and EG, respectively $(\mathrm{P}>0.05)$. Mean AFS scores preoperative were $8.48 \pm 1.73,8.74 \pm 1.19$, and $8.21 \pm 1.64$ for $P G$, TG, and EG, respectively $(\mathrm{P}>0.05)$. Additionally, there were no statistically significant differences between the groups in terms of pregnancy history, uterine cavity operations, and degree of adhesion $(\mathrm{P}>0.05)$, which means the two groups were comparable (details in Table 1).

\section{Surgical efficacy}

Mean AFS scores postoperative were $2.53 \pm 1.07,3.17 \pm 1.45$, and $3.32 \pm 1.29$ for PG, TG, and EG, respectively. In all three groups, AFS scores decreased significantly. Furthermore, the AFS scores of PGs in postoperative were significantly lower than TG and EG $(\mathrm{P}<0.05)$. Most patients' shape of the uterine cavity returned to normal, which was not statistically significant $(\mathrm{P}>0.05)$. During a 24-month post-surgery period, the pregnancy rate of $\mathrm{PG}$ was $71.6 \%$, which was significantly higher than that of TG (47.7\%) and EG (41.1\%), and all of them conceived naturally. Importantly, the live birth rate of PG was $56.8 \%$, and this was significantly different in comparison with TG (38.1\%) and EG (23.2\%). Finally, the spontaneous miscarriages of PG were the lowest, while EG was the highest, which was statistically significant $(\mathrm{P}<0.05)$ (Table 2).

\section{Surgical safety and feasibility}

No surgical complications (such as uterine perforations, moderate or severe fluid overload, heavy uterine bleeding) 
Table 2 Safety, feasibility and postoperative efficacy of three different hysteroscopic techniques

\begin{tabular}{|c|c|c|c|c|c|}
\hline Variable & $P G(n=81)$ & $\mathrm{TG}(\mathrm{n}=42)$ & $E G(n=56)$ & P1 (PG vs. TG) & P2 (PG vs. EG) \\
\hline AFS reduction (mean $\pm \mathrm{SD}$ ) & $5.85(1.85)$ & $5.60(1.52)$ & $4.89(1.97)$ & 0.441 & $0.004^{*}$ \\
\hline Pregnancy outcome, n (\%) & & & & $0.039^{*}$ & $<0.001^{*}$ \\
\hline Spontaneous miscarriages & $8(9.9)$ & $4(9.5)$ & $9(16.1)$ & & \\
\hline Live birth & $46(56.8)$ & $16(38.1)$ & $13(23.2)$ & & \\
\hline Infertility & $23(28.4)$ & $22(52.4)$ & $33(58.9)$ & & \\
\hline Surgical complications & 0 & 0 & 0 & - & - \\
\hline Surgical technique replacement & 0 & 0 & 0 & - & - \\
\hline
\end{tabular}

*, significant difference. PG, ploughing group; TG, traditional group; EG, electrosurgical group.

and no surgical technique exchanges (for example, cold scissors transfer to use a resectoscope or resectoscope transfer to use cold scissors) were recorded.

\section{Discussion}

Since hysteroscopy has emerged, IUA surgical methods have gradually changed from the traditional blind uterine cavity dilation and curettage to HA. In general, there are two different instruments used in HA: energy instruments and non-energy instruments. Energy instruments include L-hook electrodes, loop electrodes, or lasers, while nonenergy instruments mainly refer to cold scissors. Energy instruments quickly dissect cohesive adhesions or uterine cavity scars that present in most of moderate or severe IUA patients. They convert electricity into heat to achieve local cutting or electrocoagulation. A majority of currently published papers report $(13,14)$ that HA using electrical instruments for IUA patients may cause thermal injury to the endometrium. Surgeons who prefer the nonenergy instruments insist that cold scissors do not release heat and that there is no possibility of thermal damage to the endometrium adjacent to the scars. Thus, uterine cavity adhesion tissue should be separated and released to restore the uterine cavity by cold scissors. There are two contending methods that can deal with the scar tissue on the intrauterine cavity surface: cutting the scar into several parts using a "ploughing" technique; or leaving the scar tissue alone. Surgeons who prefer ploughing the scars argue that the scar tissue on the surface of the intrauterine cavity is a critical issue, as it might block the blood supply to the endometrium and cause the uterine cavity to shrink (15). However, no clinical study has been carried out to support this hypothesis. These three surgical techniques were compared through retrospective analysis of their basic preoperative features and postoperative efficacy, feasibility, and safety.

Firstly, in our study, these three groups had comparability preoperative, as there were no statistical differences in terms of age, AFS scores, pregnancy history, uterine cavity operations; the only difference between these three groups was HA technique.

After surgery, there were no complications, and the exchange of surgical techniques occurred as all hysteroscopies were done by experienced experts. The AFS scores of PGs were significantly lower than those of either EG $(\mathrm{P}<0.001)$ or TG $(\mathrm{P}=0.007)$ postoperatively. One possible reason for the higher AFS scores of EG postsurgery was that the energy-based equipment might cause damage to the endometrium by increasing inflammatory cytokines and promoting adhesion-causing agents such as transforming growth factor-beta, platelet-derived growth factor, and fibroblast growth factor after the operation (16-22). One reason for higher AFS scores of TG postsurgery was that adhesions returned more easily, as TG dissected the adhesion without dealing with the scar tissue, which retained cytokines of adhesion formation (23). Liu et al. (24) demonstrated that compared to normal endometrium, two ADAM family members of adhesion formation, ADAM-15, and ADAM-17, had increased in adhesive band tissue. Another reason was that endometrium had no space to grow and repair as the scar tissue 
undisposed. The advantage of PG was that ploughing technique opened the uterine cavity by loosening the walls from the contraction of the scarred tissue. The endometrial tissue lying under or near the scars became exposed, and the endometrium had space to grow and repair with the stimulation of postoperative estradiol valerate and progesterone. At the same time, the ploughed scar tissue strip might contract by itself and further expose more uterine cavity surface that is not covered by scar tissue.

In follow-up pregnancies within 2 years after initial surgery, PG pregnancy rates and live birth rates were significantly higher than those of either TG or EG. Possible reasons for this difference was that EG injured the endometrium and reduced the amount of residually functioning endometrium, which negatively affected implantation and the long period preceding it when a nonattached conceptus took sustenance entirely from the endometrial gland exocrine secretions (13). This was consistent with the higher abortion rates in EG than those found in PG in our study. As for TG, it separated the adhesions without dealing with scar tissue, which decreased revascularization and angiogenesis and increased expression of adhesion-related cytokines (3). This inhibited invasive trophoblast phenotype differentiation $(25,26)$. For PG, the ploughing technique allowed the myometrium blood supply to spread upwards to the endometrium and improved endometrium blood flow (15). Endometrial volume and blood flow parameters remained consistent, which was known to be important for embryo invasion and placentation (27). Furthermore, removing uterine contracture by scar ploughing could increase uterine cavity volume and promote endometrium restoration, which benefited implantation along with the growth and development of fertilized ova. The previous study (28) reported that a uterine cavity volume decrease might result in a delivery rate difference.

In this study, no uterine perforations, apparent fluid overload, or other surgical complications were recorded, and no patients had temporary surgical method change. This suggests that the cold scissor ploughing technique is a safe and feasible HA method. As the cold scissor technique has already widely been used, technical improvement needs to be made based on the cold scissor technique so it can be mastered more quickly and easily by surgeons.

In conclusion, HA with cold scissor ploughing technique was able to significantly reduce IUA AFS scores postoperatively and increase pregnancy and live birth rates when compared to either TG or EG. Also, it was feasible, safe, and easy to master for surgeons. Therefore, HA deserves to be promoted and applied in the clinic and is worthy of future prospective research.

\section{Acknowledgments}

Funding: This study is supported by National Key Research and Development Program of China (Grant No. 2018YFC1004800), Natural Science Foundation of China (Grant No. 81671492), the Hunan Science and Technology Department (Grant No. 2018SK2102) and Hunan Education Department (Grant No. XJK011CGD001).

\section{Footnote}

Conflicts of Interest: The authors have no conflicts of interest to declare.

Ethical Statement: The authors are accountable for all aspects of the work in ensuring that questions related to the accuracy or integrity of any part of the work are appropriately investigated and resolved. Approval was given to the study by The Institutional Review Board (IRB) of Third Xiangya Hospital and Xiangya Hospital, Central South University. The procedure was performed in accordance with relevant guidelines and regulations. Informed consent was obtained after the procedure was fully explained by all participants and their legal guardians.

\section{References}

1. Asherman JG. Amenorrhoea traumatica (atretica). J Obstet Gynaecol Br Emp 1948;55:23-30.

2. Sugimoto O. Diagnostic and therapeutic hysteroscopy for traumatic intrauterine adhesions. Am J Obstet Gynecol 1978;131:539-47.

3. Shokeir TA, Fawzy M, Tatongy M. The nature of intrauterine adhesions following reproductive hysteroscopic surgery as determined by early and late follow-up hysteroscopy: clinical implications. Arch Gynecol Obstet 2008;277:423-427.

4. Gan L, Duan H, Xu Q, et al. Human amniotic mesenchymal stromal cell transplantation improves endometrial regeneration in rodent models of intrauterine adhesions. Cytotherapy 2017;19:603-16.

5. Salma U, Xue M, Md Sayed AS, et al. Efficacy of intrauterine device in the treatment of intrauterine adhesions. Biomed Res Int 2014;2014:589296. 
6. Chen Y, Liu L, Luo Y, et al. Effects of Aspirin and Intrauterine Balloon on Endometrial Repair and Reproductive Prognosis in Patients with Severe Intrauterine Adhesion: A Prospective Cohort Study. Biomed Res Int 2017;2017:8526104.

7. Tan IF, Robertson M. The role of imaging in the investigation of Asherman's syndrome. Australas J Ultrasound Med 2011;14:15-8.

8. Yamamoto N, Takeuchi R, Izuchi D, et al. Hysteroscopic adhesiolysis for patients with Asherman's syndrome: menstrual and fertility outcomes. Reprod Med Biol 2013;12:159-66.

9. March CM. Management of Asherman's syndrome. Reproductive Biomedicine Online 2011;23:63-76.

10. Di Spiezio Sardo A, Calagna G, et al. Prevention of intrauterine post-surgical adhesions in hysteroscopy. A systematic review. Eur J Obstet Gynecol Reprod Biol 2016;203:182-92.

11. Zhang A, Jamail G, Xue M, et al. Hysteroscopic Intrauterine Adhesiolysis Using the "Ploughing" Technique With Cold Scissors. J Minim Invasive Gynecol 2015;22:934-5.

12. Lin XN, Zhou F, Wei ML, et al. Randomized, controlled trial comparing the efficacy of intrauterine balloon and intrauterine contraceptive device in the prevention of adhesion reformation after hysteroscopic adhesiolysis. Fertil Steril 2015;104:235-40.

13. Malhotra N, Bahadur A, Kalaivani M, et al. Changes in endometrial receptivity in women with Asherman's syndrome undergoing hysteroscopic adhesiolysis. Arch Gynecol Obstet 2012;286:525-30.

14. AAGL Elevating Gynecologic Surgery. AAGL practice report: practice guidelines on intrauterine adhesions developed in collaboration with the European Society of Gynaecological Endoscopy (ESGE). Gynecol Surg 2017;14:6.

15. Zhu K, Jamail G, Xu D, et al. Hysteroscopic Intrauterine Adhesiolysis Using "Ploughing" Technique With Cold Scissors. J Minim Invasive Gynecol 2015;22:S122.

16. Marchi E, Vargas FS, Takemura RL, et al. Monoclonal antibodies anti-TGF $\beta 1$ and anti-VEGF inhibit the experimental pleurodesis induced by silver nitrate. Growth Factors 2012;30:304-9.

17. Cheong YC, Laird SM, Li TC, et al. Peritoneal healing and adhesion formation/reformation. Hum Reprod Update 2001;7:556-66.

18. Holmdahl L, Kotseos K, Bergström M, et al. Overproduction of transforming growth factor-beta 1
(TGF-beta1) is associated with adhesion formation and peritoneal fibrinolytic impairment. Surgery 2001;129:626-32.

19. Chegini N, Rong H, Bennett B, et al. Peritoneal fluid cytokine and eicosanoid levels and their relation to the incidence of peritoneal adhesion. J Soc Gynecol Investig 1999;6:153-7.

20. Ghellai AM, Stucchi AF, Chegini N, et al. Role of transforming growth factor beta-1 in peritonitis-induced adhesions. J Gastrointest Surg 2000;4:316-23.

21. Freeman ML, Saed GM, Elhammady EF, et al. Expression of transforming growth factor beta isoform mRNA in injured peritoneum that healed with adhesions and without adhesions and in uninjured peritoneum. Fertil Steril 2003, 80 Suppl 2:708-13.

22. Minaev SV, Obozin VS, Barnash GM, et al. The influence of enzymes on adhesive processes in the abdominal cavity. Eur J Pediatr Surg 2009;19:380-3.

23. Evans-Hoeker EA, Young SL. Endometrial receptivity and intrauterine adhesive disease. Semin Reprod Med 2014, 32:392-401.

24. Liu D, Ha C, Zhang X, et al. Molecular implication of ADAM-15 and -17 in intrauterine adhesions. Eur J Obstet Gynecol Reprod Biol 2013;170:264-9.

25. Janatpour MJ, McMaster MT, Genbacev O, et al. Id-2 regulates critical aspects of human cytotrophoblast differentiation, invasion and migration. Development 2000;127:549-58.

26. Caniggia I, Mostachfi H, Winter J, et al. Hypoxiainducible factor- 1 mediates the biological effects of oxygen on human trophoblast differentiation through TGFbeta(3). J Clin Invest 2000;105:577-87.

27. Ni J, Han B, Liang J, et al. Three-dimensional 3D ultrasound combined with power Doppler for the differential diagnosis of endometrial lesions among infertile women. Int J Gynaecol Obstet 2019;145:212-8.

28. Gao H, Liu DE, Li Y, et al. Uterine size and volume are associated with a higher clinical pregnancy rate in patients undergoing assisted reproduction technology: A longitudinal study (A STROBE-compliant article). Medicine (Baltimore) 2019;98:e14366.

Cite this article as: Zhao X, Zhang A, Gao B, Burjoo A, Huang $\mathrm{H}, \mathrm{Xu}$ D. Cold scissors ploughing technique in hysteroscopic adhesiolysis: a comparative study. Ann Transl Med 2020;8(4):50. doi: 10.21037/atm.2019.11.136 